\title{
Aplicación del gel de alendronato al $1 \%$ como coadyuvante en la terapia periodontal
}

\author{
Application of $1 \%$ alendronate gel as an adjuvant \\ in periodontal therapy
}

Cristina Franshesca Franco Iglesias ${ }^{1 a}$ Ivan Zapata Tello ${ }^{1 b}$

(D) https://orcid.org/0000-0002-0388-2171

(D) https://orcid.org/0000-0003-2387-8329

Correspondencia: franshescafrancoiglesias@gmail.com

\section{Resumen}

La periodontitis es una de las afecciones más comunes que afectan a la cavidad bucal, cuya técnica de tratamiento más frecuente es el raspado y alisado radicular; sin embargo, esta técnica es considerada por varios estudios insuficiente para el tratamiento de la periodontitis, es así que se propone la aplicación del alendronato al $1 \%$ por su efecto de inhibir la producción de interleukina-1a (IL), IL-6 y TNF y ayudar a la neoformación ósea. Su efecto por vía oral nos muestra un alentador resultado de regeneración ósea, tal como se muestra en este estudio, pero, además, varios estudios mencionados en este trabajo muestran un mejor resultado aplicándolo en forma de gel al $1 \%$ y de manera local como coadyuvante de la terapia mecánica. Conclusión: El alendronato al $1 \%$, según la literatura consultada, nos muestra prometedores resultados en una aplicación local.

Palabras clave: periodontitis, alendronato, recesión gingival, gingivitis

\section{Abstract}

Periodontitis is one of the most common conditions that affect the oral cavity, whose most frequent treatment technique is scraping and root planing. However, according to several studies, this technique is considered insufficient for the treatment of periodontitis, so the application of $1 \%$ alendronate for its effect of inhibiting the production of interleukin-1a (IL), IL-6 and TNF and help new bone formation. Its effect orally shows us an encouraging result of bone regeneration, as shown in this study, but also several studies mentioned in this work show a better result applying it in the form of a $1 \%$ gel and locally as an adjuvant to the mechanical therapy. Conclusion: alendronate 1 $\%$, according to the literature consulted, shows promising results in a local application.

Keywords: periodontitis, alendronate, gingival recession, gingivitis

\footnotetext{
${ }^{1}$ Universidad Alas Peruanas (sucursal). Escuela Profesional de Estomatología. Tacna, Perú

a Estudiante

${ }^{\mathrm{b}}$ Docente universitario. Cirujano dentista
} 


\section{Introducción}

La periodontitis es la segunda enfermedad oral más frecuente en la población, tratándose de una patología inmunoinflamatoria que causa muchas veces la pérdida de piezas dentales. ${ }^{1}$

El raspado y alisado radicular (RAR) es el tratamiento tradicional y más usado para el control de esta patogenia. El objetivo del RAR es poder eliminar los cálculos de las superficies dentales para así poder reducir la carga bacteriana e inhibir la progresión inflamatoria; sin embargo, la mayoría de veces, este tratamiento no siempre es suficiente, es por eso que numerosos estudios han propuesto el uso de tratamientos coadyuvantes para complementar el RAR y así poder controlar mejor a los pacientes que padecen esta patología y evitar la pérdida de las piezas dentales.

El alendronato es un bifosfonato usado en tratamientos para la osteoporosis. Este fármaco actúa inhibiendo la reabsorción ósea e induce a los osteoblastos a promover formación ósea temprana. ${ }^{2}$

Para esta revisión se realizó una búsqueda de artículos científicos en las bases de datos PubMed, Scielo, Journal Periodontology, Journal of Oral Science y buscadores académicos utilizando como palabras clave: alendronatos, bifosfonatos, periodontitis, enfermedad periodontal, gel.

Se restringió la búsqueda a artículos que fueron publicados entre el 2010 al 2020 y se aceptaron artículos en cualquier idioma.

Se seleccionaron un total de 25 artículos, entre revisiones sistemáticas, ensayos clínicos aleatorizados y ensayos experimentales realizados en seres humanos y animales. Se excluyeron los artículos que no mostraban resultados.

\section{Tema central del estudio}

La periodontitis es una afección inmunoinflamatoria, la cual se caracteriza por la pérdida ósea alveolar y el soporte dentario como consecuencia de mediadores inflamatorios que actúan sobre el proceso de osteoclastogénesis. ${ }^{3}$

A lo largo del tiempo, el tratamiento para la periodontitis fue puramente mecánico y/o quirúrgico; sin embargo, en la actualidad, se están buscando tratamientos alternativos que contribuyan a la regeneración ósea guiada. $^{4-6}$

Una de estas alternativas, como coadyuvante, es el uso de bifosfonatos, destacando el alendronato, el cual se ha comprobado que inhibe la producción de interleukina-1a (IL), IL-6 y TNF causada por los monocitos y la reabsorción ósea, desencadenando propiedades osteoestimulativas. ${ }^{7-9}$

En un estudio realizado en las diáfisis del fémur del ratón, la aplicación directa de alendronato aceleró y promovió, localmente, la regeneración ósea, comportándose como material osteoinductivo; además mejoró la calidad de tejido óseo neoformado y la densidad del tejido óseo. Se corroboraron los resultados radiográficamente. ${ }^{8}$

Lane ${ }^{10}$ utilizó bifosfonatos por vía oral como complemento de la terapia periodontal, este estudio nos muestra como resultado una mejora de la inserción clínica y la profundidad de sondaje en los pacientes que utilizaron los bifosfonatos orales; por otro lado, Rocha, en sus estudios, nos muestra que administrando $10 \mathrm{mg}$ al día 
de alendronato por vía oral se observa que, después de 6 meses de uso, la altura de la cresta ósea alveolar mejoró significativamente. ${ }^{11,12}$

Con toda esta información, diversos investigadores realizaron la aplicación subgingival de gel de alendronato al $1 \%$, esto después de realizar la mecanoterapia.

De 25 artículos seleccionados para esta revisión, 12 son ensayos clínicos; de los cuales, 8 se realizaron utilizando sitios controles y sitios experimentales. En los sitios experimentales se vio una profundidad de sondaje media disminuida $(1.65 \pm 1.35 \mathrm{~mm})$, una ganancia horizontal y vertical de inserción clínica $(3.27 \pm 1.11 \mathrm{~mm})$ y diferencias significativas en el relleno óseo de los sitios experimentales.

En el estudio de Sharma y Pradeep, el gel de alendronato al $1 \%$ aplicado (10 $\mathrm{mg})$ después de realizada la mecanoterapia, una vez al mes (durante 6 meses), nos muestra una mejora en la ganancia del nivel de inserción clínica y relleno óseo. ${ }^{7,13,14}$ En su estudio, Dutra nos muestra que el suministro local de alendronato al $1 \%$, en una lesión de furca de Clase II, el nivel de inserción clínica vertical y horizontal es favorable y presenta también una mejora en el relleno óseo. ${ }^{15}$

También existen estudios donde se aplica el gel de alendronato al $1 \%$ en pacientes que tienen mayor predisposición de adquirir periodontitis; tal es el caso de pacientes fumadores y con diabetes, resultando una ganancia del nivel de inserción clínica, reducción de la profundidad de sondaje y una ganancia significativa en la altura de la cresta alveolar. Se encontró un porcentaje medio significativamente mayor de relleno óseo en el grupo de ALN (41.05 $\pm 11.40 \%)$ en comparación con el grupo de placebo $(2.5 \pm 0.93 \%) .4,16,17$

Si se compara el gel de alendronato al $1 \%$ con otros medicamentos subgingivales como son el gel de áloe vera y atorvastatina al $1.2 \%$ y el plasma rico en fibrina, el gel de alendronato al $1 \%$ demuestra mayor eficacia como coadyuvante del tratamiento periodontal. ${ }^{5,18,19}$

El uso combinado de gel de alendronato al $1 \%$ con plasma rico en fibrina nos muestra un efecto potencializador, exhibiendo mejores resultados clínicos y radiográficos, sin ningún efecto adverso. ${ }^{20,21}$

Se puede observar en varios autores, que se obtuvieron resultados positivos tras el uso de alendronato; sin embargo, es de conocimiento que existen algunos casos que pueden desarrollar osteonecrosis mandibular, siendo este el efecto secundario más perjudicial, ${ }^{22-24}$ pero la incidencia de que esto suceda es baja. Un estudio realizado por Duriet en el 2005 nos muestra que de 1232 pacientes que se tratan con bifosfonatos por vía oral o endovenosa, solo 64 fueron diagnosticados con osteonecrosis de la mandíbula. ${ }^{25}$

\section{Conclusiones}

Los estudios presentados en esta revisión de literatura nos muestran unos resultados muy prometedores del gel de alendronato al $1 \%$ para su aplicación en la terapia de la enfermedad periodontal, mejorando el nivel de inserción clínica, disminuyendo la profundidad de sondaje y contribuyendo a la regeneración ósea de la cresta alveolar. Es así que el uso de este gel como coadyuvante de la terapia periodontal podría darnos un mejor pronóstico de la periodontitis, mejorando la salud bucal de nuestros pacientes y a su vez la calidad de vida de los mismos. 


\section{Recomendaciones}

En comparación con otros tratamientos, el gel de alendronato al $1 \%$ aún no tiene un protocolo de tratamiento en el que concuerden los autores; sin embargo, este estudio puede ayudar a continuar con la investigación del uso del gel de alendronato al $1 \%$ de manera tópica con casos controles, de esa manera se podría trabajar en una mayor población y evaluar también los posibles efectos secundarios.

\section{Referencias}

1. Quesada Llanto IM. Prevalencia de la enfermedad periodontal en pacientes atendidos en la Universidad Peruana Los Andes 2016. Universidad Peruana Los Andes; 2018. Disponible en: http://repositorio.upla. edu.pe/handle/UPLA/327

2. Giger E V., Castagner B. Biomedical applications of bisphosphonates. Vol. 167, Journal of Controlled Release. 2013. p. 175-88.

3. Tavares SJ da S, Menezes AD. Uso dos bifosfonatos como coadjuvantes na terapia periodontal - revisão de literatura. Rev Interfaces Saúde. 2017;4(12):41-50.

4. Sheokand V, Chadha VS. The comparative evaluation of $1 \%$ alendronate gel as local drug delivery system in chronic periodontitis in smokers and non smokers: Randomized clinical trial. J Oral Biol Craniofacial Res. 2019; 9(2):198-203.

5. Ipshita S, Kurian IG. One percent alendronate and aloe vera gel local host modulating agents in chronic periodontitis patients with class II furcation defects: A randomized, controlled clinical trial. J Investig Clin Dent. 2018; 9(3):e12334. Disponible en: https://onlinelibrary.wiley.com/doi/abs/10.1111/jicd.12334

6. Akram Z, Abduljabbar T. Efficacy of bisphosphonate as an adjunct to nonsurgical periodontal therapy in the management of periodontal disease: a systematic review. Vol. 83, British Journal of Clinical Pharmacology. 2017. Disponible en: https://pubmed.ncbi.nlm.nih.gov/27718252/

7. Sharma A, Pradeep AR. Clinical Efficacy of $1 \%$ Alendronate Gel as a Local Drug Delivery System in the Treatment of Chronic Periodontitis: A Randomized, Controlled Clinical Trial. J Periodontol. 2012; 83(1):11-8. Disponible en: http://doi.wiley.com/10.1902/jop.2011.110091

8. Gait MT, Fontana S. Efecto del alendronato sobre la remodelación del tejido óseo. Estudio experimental. claves en Odontol. 2010;(68):9-19.

9. Silva RAB, Sousa $\square$ Pereira AP. Alendronate inhibits osteocyte apoptosis and inflammation via IL $\square 6$, inhibiting bone resorption in periapical lesions of ovariectomized rats. Int Endod J. 2020; 53(1):84-96. Disponible en: https://onlinelibrary.wiley.com/doi/abs/10.1111/iej.13206

10. Lane N, Armitage GC. Bisphosphonate therapy improves the outcome of conventional periodontal treatment: results of a 12-month, randomized, placebo-controlled study. J Periodontol. 2005; 76(7):111322. Disponible en: https://pubmed.ncbi.nlm.nih.gov/16018754/

11. Rocha ML, Malacara JM. Effect of alendronate on periodontal disease in postmenopausal women: a randomized placebo-controlled trial. J Periodontol. 2004; 75(12):1579-85. Disponible en: https://pubmed. ncbi.nlm.nih.gov/15732857/

12. Rocha M, Nava LE. Clinical and radiological improvement of periodontal disease in patients with type 2 diabetes mellitus treated with alendronate: a randomized, placebo-controlled trial. J Periodontol. 2001; 72(2):204-9. Disponible en: https://pubmed.ncbi.nlm.nih.gov/11288794/

13. Sharma A, Pradeep AR. Clinical efficacy of $1 \%$ alendronate gel in adjunct to mechanotherapy in the treatment of aggressive periodontitis: a randomized controlled clinical trial. J Periodontol. 2012; 83(1):1926. Disponible en: http://doi.wiley.com/10.1902/jop.2011.110206

14. Dutra BC, Oliveira AMSD. Effect of $1 \%$ sodium alendronate in the non-surgical treatment of periodontal intraosseous defects: A 6-month clinical trial. J Appl Oral Sci. 2017;25(3):310-7. 
15. Pradeep AR, Kumari M. $1 \%$ Alendronate gel as local drug delivery in the treatment of class ii furcation defects: a randomized controlled clinical trial. J Periodontol; 84(3):307-15. Disponible en: http://doi.wiley. com/10.1902/jop.2012.110729

16. Sharma A, Raman A, Pradeep AR. Role of $1 \%$ alendronate gel as adjunct to mechanical therapy in the treatment of chronic periodontitis among smokers. J Appl Oral Sci. 2017; 25(3):243-9. Disponible en: https://pubmed.ncbi.nlm.nih.gov/28678942/

17. Pradeep AR, Sharma A. Local drug delivery of alendronate gel for the treatment of patients with chronic periodontitis with diabetes mellitus: a double-masked controlled clinical trial. J Periodontol. 2012; 83(10):1322-8. Disponible en: http://doi.wiley.com/10.1902/jop.2012.110292

18. Pradeep AR, Kanoriya D. Chatterjee A. Comparative evaluation of subgingivally delivered $1 \%$ alendronate versus $1.2 \%$ atorvastatin gel in treatment of chronic periodontitis: a randomized placebo-controlled clinical trial. J Investig Clin Dent. 2017; 8(3):e12215. Disponible en: http://doi.wiley.com/10.1111/jicd.12215

19. Wanikar I, Rathod S. Clinico-radiographic evaluation of $1 \%$ alendronate gel as an adjunct and smart blood derivative platelet rich fibrin in grade II furcation defects. J Periodontol. 2019; 90(1):52-60. Disponible en: http://doi.wiley.com/10.1002/JPER.18-0146

20. Kanoriya D, Pradeep AR. Mandibular degree ii furcation defects treatment with platelet-rich fibrin and $1 \%$ alendronate gel combination: a randomized controlled clinical trial. J Periodontol. 2017 (3):250-8. Disponible en: http://doi.wiley.com/10.1902/jop.2016.160269

21. Li F, Jiang P, Pan J. Synergistic application of platelet-rich fibrin and $1 \%$ alendronate in periodontal bone regeneration:Ameta-analysis. Biomed Res Int. 2019; Disponible en: https://doi.org/10.1155/2019/9148183

22. Melo MD. Osteonecrosis of the jaws in patients with a history of receiving bisphosphonate therapy: Strategies for prevention and early recognition. J Am Dent Assoc. 2005. (12):1675-81.

23. Marx RE, Sawatari Y. Bisphosphonate-induced exposed bone (osteonecrosis/osteopetrosis) of the jaws: Risk factors, recognition, prevention, and treatment. J Oral Maxillofac Surg. 2005. 63(11):1567-75.

24. Estilo CL, Van Poznak CH. Osteonecrosis of the maxilla and mandible in patients with advanced cancer treated with bisphosphonate therapy. Oncologist. 2008;13(8):911-20.

25. Durie BG, Katz M. Osteonecrosis of the jaw and bisphosphonates. N Engl J Med 2005; 353:99-102.

- Conflicto de intereses: La presente investigación no presenta conflicto de intereses entre los investigadores.

- Fuente de financiamiento: La presente investigación fue financiada por los investigadores. 